Homology, Homotopy and Applications, vol.20(2), 2018, pp.361-375

\title{
ON THE MOTIVIC PETERSON CONJECTURE
}

\author{
MASAKI KAMEKO
}

(communicated by Martin Crossley)

\begin{abstract}
We show that the analogue of the Peterson conjecture on the action of Steenrod squares does not hold in motivic cohomology.
\end{abstract}

\section{Introduction}

The mod 2 cohomology $H^{*}\left(B V_{n}\right)$ of the classifying space of the elementary abelian 2 -group $V_{n}=(\mathbb{Z} / 2)^{n}$ of rank $n$ is a polynomial algebra of $n$-variables

$$
\mathbb{Z} / 2\left[x_{1}, \ldots, x_{n}\right]
$$

where $\operatorname{deg} x_{i}=1$. We denote it by $P_{n}$. Also, we denote by $P_{n}^{d}$ the subspace of $P_{n}$ spanned by monomials of degree $d$. Then, we have

$$
P_{n}=\bigoplus_{d=0}^{\infty} P_{n}^{d}
$$

Throughout this paper, an element means a homogeneous element. The action of the Steenrod squares $\mathrm{Sq}^{1}, \mathrm{Sq}^{2}, \mathrm{Sq}^{3}, \ldots$ on $P_{n}$ is given by the unstable condition

$$
\begin{aligned}
& \operatorname{Sq}^{1}\left(x_{i}\right)=x_{i}^{2}, \\
& \operatorname{Sq}^{a}\left(x_{i}\right)=0 \quad \text { for } a>1,
\end{aligned}
$$

and the Cartan formula

$$
\mathrm{Sq}^{a}(x \cdot y)=\sum_{b=0}^{a} \operatorname{Sq}^{a-b}(x) \cdot \operatorname{Sq}^{b}(y),
$$

where $x, y \in P_{n}$ and $\mathrm{Sq}^{0}$ is the identity map, that is, $\mathrm{Sq}^{0}(x)=x, \mathrm{Sq}^{0}(y)=y$.

Finding a minimal set of generators of $P_{n}$ as a module over the mod 2 Steenrod algebra $\mathcal{A}$ is known as the hit problem. One may consider the quotient space

$$
Q P_{n}^{d}=P_{n}^{d} /\left(\mathcal{A}_{+}\left(P_{n}\right) \cap P_{n}^{d}\right)
$$

where $\mathcal{A}_{+}$is the set of positive degree elements in the mod 2 Steenrod algebra $\mathcal{A}$ and

$$
\mathcal{A}_{+}\left(P_{n}\right)=\left\{a x \mid a \in \mathcal{A}_{+}, x \in P_{n}\right\} .
$$

Then, the hit problem is the problem of finding a basis for $Q P_{n}^{d}$. Since its formulation in mid-1980 by Peterson through the computation of $Q P_{2}^{d}$, the hit problem has been

This work was supported by JSPS KAKENHI Grant Numbers JP25400097 and JP17K05263.

Received December 22, 2017, revised April 9, 2018; published on July 18, 2018.

2010 Mathematics Subject Classification: 55S10, 14F42.

Key words and phrases: Steenrod algebra, motivic cohomology, hit problem.

Article available at http://dx.doi.org/10.4310/HHA.2018.v20.n2.a18

Copyright (C) 2018, International Press. Permission to copy for private use granted. 
and is studied by many mathematicians. Among recently published papers and books are Ault [1], Pengelley and Williams [4], and Sum [6] and Walker and Wood [8].

The most fundamental result on the hit problem is Wood's theorem. It was known as Peterson's conjecture before Wood proved it. The Peterson conjecture was formulated in terms of the number $\alpha(d+n)$ of 1's in the binary expression of $d+n$. However, we may state it in terms of the function $\beta(d)$ defined by

$$
\beta(d)=\min \left\{s \in \mathbb{N} \mid d=\left(2^{i_{1}}-1\right)+\cdots+\left(2^{i_{s}}-1\right), i_{1}, \ldots, i_{s} \in \mathbb{N}\right\} .
$$

Theorem $1.1($ Wood $[\mathbf{9}])$. If $\beta(d)>n$, then $\operatorname{dim} Q P_{n}^{d}=0$.

Wood's theorem gives us a sufficient condition for $Q P_{n}^{d}=\{0\}$ in terms of $d$ and $n$. The condition $\beta(d)>n$ is also a necessary condition for $Q P_{n}^{d}=\{0\}$ since a monomial of the form

$$
x_{1}^{2^{i_{1}}-1} \cdots x_{n}^{2^{i_{n}}-1}
$$

is not in $\mathcal{A}_{+}\left(P_{n}\right)$. Furthermore, Wood's theorem is the foundation for various results on the hit problem, for example, the computation of $\operatorname{dim} Q P_{3}^{d}$ and so on. Singer's transfer homomorphism [5] relates the hit problem to the cohomology of the mod 2 Steenrod algebra. It is the $E_{2}$-term of the classical Adams spectral sequence hence the hit problem is related to the stable homotopy theory. Notably, Minami's new doomsday conjecture [3] is inspired by the Peterson conjecture and its consequences.

On the other hand, in the $21^{\text {st }}$ century, motivic cohomology theory is studied in both algebraic geometry and algebraic topology. In particular, a motivic analogue of the Adams spectral sequence and its $E_{2}$-term, that is, the cohomology of the mod 2 motivic Steenrod algebra, is studied by Dugger and Isaksen [2]. So, it is reasonable to think of motivic counterparts of the hit problem, Singer transfer, new doomsday conjecture and so on.

In this paper, we consider the hit problem in motivic cohomology and an analogue of the Peterson conjecture. To be precise, we disprove the motivic version of the Peterson conjecture. Our result seems to indicate a significant difference between the classical stable homotopy theory and the motivic stable homotopy theory. We hope our result sheds some light on both classical and motivic stable homotopy theory.

For simplicity, we assume that the base field is the complex number field. Then, $H^{*, *}(\operatorname{Spec}(\mathbb{C}))=\mathbb{Z} / 2[\tau]$, where $\operatorname{deg} \tau=(0,1)$. We refer the reader to [7, Section 9] and [10, Section 2] for the details of the mod $2 \operatorname{motivic}$ Steenrod algebra $\mathcal{A}^{* * *}$ and the mod 2 motivic cohomology $H^{*, *}\left(B V_{n}\right)$ of $B V_{n}$. The mod 2 motivic cohomology of the classifying space of elementary abelian 2-group $V_{n}$ of rank $n$ is given by

$$
M_{n}=\mathbb{Z} / 2\left[\tau, x_{1}, \ldots, x_{n}, y_{1}, \ldots, y_{n}\right] /\left(x_{1}^{2}+\tau y_{1}, \ldots, x_{n}^{2}+\tau y_{n}\right),
$$

where $\operatorname{deg} \tau=(0,1), \operatorname{deg} x_{i}=(1,1), \operatorname{deg} y_{i}=(2,1)$. The mod 2 motivic Steenrod algebra is generated by $Q_{0}, \wp^{1}, \wp^{2}, \wp^{3}, \ldots$ Its action on the $M_{n}$ is given by the unstable condition

$$
\begin{aligned}
& Q_{0}(\tau)=0, \quad \wp^{a}(\tau)=0 \quad \text { for } a \geqslant 1, \\
& Q_{0}\left(x_{i}\right)=y_{i}, \quad \wp^{a}\left(x_{i}\right)=0 \quad \text { for } a \geqslant 1 \text {, } \\
& Q_{0}\left(y_{i}\right)=0 \quad \wp^{1}\left(y_{i}\right)=y_{i}^{2}, \quad \wp^{a}\left(y_{i}\right)=0 \quad \text { for } a \geqslant 2 \text {, }
\end{aligned}
$$


and the Cartan formula

$$
\begin{aligned}
& Q_{0}(x y)=Q_{0}(x) y+x Q_{0}(y), \\
& \wp^{a}(x y)=\sum_{b=0}^{a} \wp^{a-b}(x) \wp^{b}(y)+\tau \sum_{b=0}^{a-1} Q_{0} \wp^{a-1-b}(x) Q_{0} \wp^{b}(y) .
\end{aligned}
$$

The mod 2 Steenrod algebra is given as the quotient of the mod 2 motivic Steenrod algebra, that is, $\mathcal{A}=\mathcal{A}^{* * *} /(\tau+1)$. Similarly, we have $M_{n} /(\tau+1)=P_{n}$ and the projection

$$
M_{n} \rightarrow P_{n}
$$

sending $\tau, x_{i}, y_{i}$ to $1, x_{i}, x_{i}^{2}$, respectively. This projection is nothing but the realization map

$$
H^{*, *}\left(B V_{n}\right) \rightarrow H^{*}\left(B V_{n}\right) .
$$

We denote by $\mathcal{A}_{+}^{*, *}$ the set of elements $a$ in $\mathcal{A}^{*, *}$ such that the sum of the first and second degrees of $a$ is positive and let

$$
\mathcal{A}_{+}^{*, *}\left(M_{n}\right)=\left\{a x \mid a \in \mathcal{A}_{+}^{*, *}, x \in M_{n}\right\} .
$$

Let $M_{n}^{d, *}$ be the subspace of $M_{n}$ spanned by elements of degree $(d, *)$. We define $Q M_{n}^{d, *}$ by

$$
Q M_{n}^{d, *}=M_{n}^{d, *} /\left(\mathcal{A}_{+}^{*, *}\left(M_{n}\right) \cap M_{n}^{d, *}\right) .
$$

We call the problem of finding a minimal set of generators of $M_{n}$ as a module over the mod 2 motivic Steenrod algebra $\mathcal{A}^{*, *}$ the motivic hit problem. It is equivalent to the problem of finding a basis for $Q M_{n}^{d, *}$. A monomial of the form

$$
x_{1} \cdots x_{n} y_{1}^{2^{i_{1}-1}-1} \cdots y_{n}^{2^{i_{n}-1}-1}
$$

in $M_{n}^{d, *}$ is not in $\mathcal{A}_{+}^{*, *}\left(M_{n}\right)$. So, if $\beta(d) \leqslant n, \operatorname{dim} Q M_{n}^{d, *} \neq 0$. Thus, it is reasonable to ask the following conjecture.

Conjecture 1.2. If $\beta(d)>n$, then $\operatorname{dim} Q M_{n}^{d, *}=0$.

This conjecture holds for some $n$ and this is the motivic analogue of the Peterson conjecture. The purpose of this paper is to give counterexamples for this conjecture.

Theorem 1.3. For $n$ such that $\alpha(n-2) \geqslant 3$, let $k=n-3, d=(n-1)\left(2^{k+1}-2\right)+k$. Then, $\beta(d)>n$ but $\operatorname{dim} Q M_{n}^{d, *} \neq 0$.

For $n=9$, the assumption of Theorem 1.3 holds. For $n=9$, we have $k=6, d=$ 1014 and $\beta(1014)=10>9$. We prove Theorem 1.3 by giving a family of monomials not in $\mathcal{A}_{+}^{*, *}\left(M_{n}\right)$. Let us denote by $z_{k}$ the monomial

$$
x_{1} \cdots x_{k} y_{1}^{2^{k-1}-1} y_{2}^{2^{k}-2^{k-2}-1} \cdots y_{k}^{2^{k}-2^{0}-1} y_{k+1}^{2^{k}-1} \cdots y_{n}^{2^{k}-1}
$$

of degree $d=(n-1)\left(2^{k+1}-2\right)+k$. Then, Theorem 1.3 could be divided to the following Propositions 1.4 and 1.5.

Proposition 1.4. Suppose that $1 \leqslant k<n$. Then, the monomial $z_{k}$ is not in $\mathcal{A}_{+}^{* * *}\left(M_{n}\right)$.

Proposition 1.5. Suppose that $\alpha(n-2) \geqslant 3$ and $k=n-3$. Then, we have $\beta(d)>n$. 
This paper is organized as follows. In Section 2, we prove Proposition 1.5. In Section 3, we recall some results on the classical hit problem. In Section 4, we give the details of the motivic hit problem. In Section 5, we prove Proposition 1.4.

\section{Acknowledgments}

The author would like to thank Nobuaki Yagita and the referee for comments and suggestions for improving the exposition of this paper.

\section{Proof of Proposition 1.5}

In this section, we prove Proposition 1.5. First, we prove Proposition 2.1 below.

Proposition 2.1. Let $d, n$ be positive integers. Then, $\alpha(d+n)>n$ if and only if $\beta(d)>n$, where $\alpha(d+n)$ is the number of 1 's in the binary expansion of $d+n$.

Proof. We prove that $\alpha(d+n) \leqslant n$ if and only if $\beta(d) \leqslant n$. Recall that

$$
\alpha(t)=\min \left\{s \mid t=2^{i_{1}}+\cdots+2^{i_{s}}, i_{1} \geqslant i_{2} \geqslant \cdots \geqslant i_{s} \geqslant 0\right\} .
$$

Therefore, we have $\alpha(s) \leqslant s$.

Suppose that $\beta(d) \leqslant n$. Then, we have

$$
d=\left(2^{i_{1}}-1\right)+\cdots+\left(2^{i_{n}}-1\right)
$$

for some $i_{1} \geqslant \cdots \geqslant i_{n} \geqslant 0$. Hence, we have

$$
d+n=2^{i_{1}}+\cdots+2^{i_{n}} .
$$

Therefore, we have $\alpha(d+n) \leqslant n$.

Suppose that $\alpha(d+n) \leqslant n$. Let $s$ be the least positive integer such that

$$
\alpha(d+s) \leqslant s .
$$

By the definition of $s$, we have $s \leqslant n$. Let $r=\alpha(d+s)$. Then, again, by the definition of $s, r \leqslant s$ and

$$
d+s=2^{i_{1}}+\cdots+2^{i_{r}}
$$

for some $i_{1}>\cdots>i_{r} \geqslant 0$. So,

$$
d+s-1=2^{i_{1}}+\cdots+2^{i_{r}}-1=2^{i_{1}}+\cdots+2^{i_{r-1}}+2^{i_{r}-1}+\cdots+2^{0}
$$

and by the definition of $s$, we have $\alpha(d+s-1)=r-1+i_{r}>s-1$. Therefore, we have $i_{r}>s-r \geqslant 0$. Let $j_{a}=i_{a}$ for $a \leqslant r-1, j_{a}=i_{r}-1-a+r$ for $r \leqslant a \leqslant s-1$ and $j_{a}=i_{r}-s+r$ for $a=s$. Then,

$$
d+s=2^{j_{1}}+\cdots+2^{j_{s}} .
$$

Hence, we have

$$
d=\left(2^{j_{1}}-1\right)+\cdots+\left(2^{j_{s}}-1\right) .
$$

Therefore, we have $\beta(d) \leqslant s \leqslant n$. 
Remark 2.2. Suppose that $d=(n-1)\left(2^{k+1}-2\right)+k$. Then, we have

$$
d+n=(n-1) \cdot 2^{k+1}+2-n+k .
$$

For $k=n-1$, we have

$$
d+n=(n-1) \cdot 2^{n}+1
$$

Hence, we have

$$
\alpha(d+n)=\alpha(n-1)+1 \leqslant n .
$$

Similarly, for $k=n-2$, we have

$$
d+n=(n-1) \cdot 2^{n-1}
$$

and

$$
\alpha(d+n)=\alpha(n-1) \leqslant n .
$$

Therefore, $\beta(d) \leqslant n$ for $k=n-1, n-2$.

Now, we prove Proposition 1.5.

Proof of Proposition 1.5. Let $d=(n-1)\left(2^{k+1}-2\right)+k$ and $k=n-3$. Then,

$$
d+n=(n-1) \cdot 2^{n-2}-1=(n-2) \cdot 2^{n-2}+2^{n-3}+\cdots+2^{0} .
$$

Hence, we have

$$
\alpha(d+n)=\alpha(n-2)+n-2 .
$$

Since we assumed that $\alpha(n-2) \geqslant 3$, we have

$$
\alpha(d+n)>n .
$$

Thus, by Proposition 2.1, we have $\beta(d)>n$.

\section{The classical hit problem}

In this section, we recall some results on the classical hit problem.

For a monomial

$$
v=x_{1}^{e_{1}} \cdots x_{n}^{e_{n}}
$$

in $P_{n}$, let us define $\alpha_{i j}(v)$ in $\{0,1\}$ by

$$
e_{i}=\sum_{j=0}^{\infty} \alpha_{i j}(v) 2^{j} .
$$

We define non-negative integers $\alpha_{i}(v), \omega_{j}(v)$ by

$$
\begin{aligned}
& \alpha_{i}(v)=\sum_{j=0}^{\infty} \alpha_{i j}(v), \\
& \omega_{j}(v)=\sum_{i=1}^{n} \alpha_{i j}(v) .
\end{aligned}
$$


For finite sequences of non-negative integers of the same length $c$, say

$$
\gamma=\left(\gamma_{1}, \ldots, \gamma_{c}\right), \quad \text { and } \quad \delta=\left(\delta_{1}, \ldots, \delta_{c}\right),
$$

we consider the lexicographic order from the left, that is, we say

$$
\gamma<\delta
$$

if and only if there exists $a \geqslant 1$ such that $\gamma_{b}=\delta_{b}$ for $b<a$ and $\gamma_{a}<\delta_{a}$.

Let $F_{n}$ be the subspace of $P_{n}$ spanned by $\mathcal{A}_{+}\left(P_{n}\right)$ and monomials $v$ in $P_{n}$ such that

$$
\left(\omega_{0}(v), \ldots, \omega_{k-1}(v)\right)<(n-1, \ldots, n-1) .
$$

We denote by $F_{n-1}$ the subspace of $P_{n-1}$ spanned by $\mathcal{A}_{+}\left(P_{n-1}\right)$ and monomials $v$ in $P_{n-1}$ such that

$$
\left(\omega_{0}(v), \ldots, \omega_{k-1}(v)\right)<(n-1, \ldots, n-1) .
$$

It is clear that $F_{n}, F_{n-1}$ are closed under the action of Steenrod squares since, for each monomial $v$ in $P_{n}^{d}$,

$$
\mathrm{Sq}^{a} v
$$

is a linear combination of monomials $w$ such that

$$
\left(\omega_{0}(w), \omega_{1}(w), \ldots\right)<\left(\omega_{0}(v), \omega_{1}(v), \ldots\right) .
$$

We denote the projections by the same symbol $\pi: P_{n} \rightarrow P_{n} / F_{n}, \pi: P_{n-1} \rightarrow P_{n-1} / F_{n-1}$.

Throughout the rest of this section, let $k, n$ be fixed positive integers and $d_{1}=$ $(n-1)\left(2^{k}-1\right)$. We consider the quotient spaces

$$
\left(P_{n} / F_{n}\right)^{d_{1}}=P_{n}^{d_{1}} /\left(F_{n} \cap P_{n}^{d_{1}}\right)
$$

and

$$
\left(P_{n-1} / F_{n-1}\right)^{d_{1}}=P_{n-1}^{d_{1}} /\left(F_{n-1} \cap P_{n-1}^{d_{1}}\right) .
$$

A monomial $v$ in $P_{n-1}^{d_{1}}$ is $x_{1}^{2^{k-1}} \cdots x_{n-1}^{2^{k}-1}$ or $\omega(v)<(n-1, \ldots, n-1)$, that is, $v \in$ $F_{n-1}$. So, it is clear that $\left(P_{n-1} / F_{n-1}\right)^{d_{1}}=\mathbb{Z} / 2$ and spanned by the single element

$$
x_{1}^{2^{k}-1} \cdots x_{n-1}^{2^{k}-1}=\pi\left(x_{1}^{2^{k}-1} \cdots x_{n-1}^{2^{k}-1}\right) .
$$

To describe a basis for the vector space $\left(P_{n} / F_{n}\right)^{d_{1}}$, we need the following definitions. For $1 \leqslant \ell \leqslant n$, let Mono $(\ell)$ be the set of monotone increasing functions

$$
\{1, \ldots, \ell\} \rightarrow\{1, \ldots, n\} .
$$

We identify $\sigma \in \operatorname{Mono}(\ell)$ with the permutation $\sigma$ on $\{1, \ldots, n\}$ such that

$$
\sigma(1)<\cdots<\sigma(\ell), \quad \sigma(\ell+1)<\cdots<\sigma(n) .
$$

Then, the permutation $\sigma$ of $\{1, \ldots, n\}$ acts on $P_{n}$ in the obvious manner, that is,

$$
\sigma\left(x_{1}^{e_{1}} \cdots x_{n}^{e_{n}}\right)=x_{\sigma(1)}^{e_{1}} \cdots x_{\sigma(n)}^{e_{n}} .
$$

For an integer $\ell$ such that $1 \leqslant \ell \leqslant \min \{k, n\}$, let us define the monomial $v_{\ell}$ in $P_{n}^{d_{1}}$ by

$$
v_{\ell}=x_{1}^{2^{\ell-1}-1} x_{2}^{2^{k}-2^{\ell-2}-1} \cdots x_{\ell}^{2^{k}-2^{0}-1} x_{\ell+1}^{2^{k}-1} \cdots x_{n}^{2^{k}-1} .
$$


First, we prove that the set of monomials

$$
\left\{\pi\left(\sigma\left(v_{\ell}\right)\right) \mid 1 \leqslant \ell \leqslant \min \{k, n\}, \sigma \in \operatorname{Mono}(\ell)\right\}
$$

spans the vectors space $\left(P_{n} / F_{n}\right)^{d_{1}}$. To this end, we prove the following Propositions 3.1 and 3.2.

Proposition 3.1. Let $v$ be a monomial in $P_{n}^{d_{1}}$. Then,

$$
\left(\omega_{0}(v), \ldots, \omega_{k-1}(v)\right) \leqslant(n-1, \ldots, n-1) .
$$

Proof. Proof by contradiction. Suppose that

$$
\left(\omega_{0}(v), \ldots, \omega_{\ell-1}(v)\right)=(n-1, \ldots, n-1)
$$

and

$$
\omega_{\ell}(v)=n
$$

for $1 \leqslant \ell<k$. Then, on the one hand, since $d_{1}$ could be written as

$$
\sum_{j=0}^{\infty} \omega_{j}(v) 2^{j-1}=(n-1)\left(2^{\ell}-1\right)+n 2^{\ell}+\sum_{j=\ell+1}^{\infty} \omega_{j}(v) 2^{j-1},
$$

we have

$$
d_{1}-(n-1)\left(2^{\ell+1}-1\right)=2^{\ell}+\omega_{\ell+1}(v) 2^{\ell+1}+\cdots .
$$

It is not divisible by $2^{\ell+1}$. On the other hand, since $d_{1}=(n-1)\left(2^{k}-1\right)$, we have

$$
d_{1}-(n-1)\left(2^{\ell+1}-1\right)=(n-1)\left(2^{k}-2^{\ell+1}\right) .
$$

It is divisible by $2^{\ell+1}$. It is a contradiction.

Proposition 3.2. For a monomial $v$ in $P_{n}^{d_{1}}$ such that

$$
\left(\omega_{0}(v), \ldots, \omega_{k-1}(v)\right)=(n-1, \ldots, n-1),
$$

there exists a unique pair $(\ell, \sigma)$ such that $1 \leqslant \ell \leqslant \min \{k, n\}, \sigma \in \operatorname{Mono}(\ell)$ and

$$
v \equiv \sigma\left(v_{\ell}\right) \quad \bmod F_{n} .
$$

Now, we prove Proposition 3.2. For each monomial $v$ in $P_{n}$, let

$$
u_{j}(v)=\left(\alpha_{1 j}(v), \ldots, \alpha_{n j}(v)\right) .
$$

Lemma 3.3. Let $v$ be a monomial such that

$$
\left(\omega_{0}(v), \ldots, \omega_{k-1}(v)\right)=(n-1, \ldots, n-1) .
$$

Suppose that $u_{j}(v)<u_{j+1}(v)$. Let $v^{\prime}$ be the unique monomial such that $u_{a}(v)=u_{a}\left(v^{\prime}\right)$ for $a \neq j, j+1$ and $u_{j}\left(v^{\prime}\right)=u_{j+1}(v), u_{j+1}\left(v^{\prime}\right)=u_{j}(v)$. Then,

$$
v \equiv v^{\prime} \bmod F_{n} .
$$


Proof. Let $w$ be the monomial such that

$$
\begin{gathered}
u_{j}(w)=(1, \ldots, 1), \\
u_{j+1}(w)=\left(\alpha_{1, j}(v) \alpha_{1, j+1}(v), \ldots, \alpha_{n, j}(v) \alpha_{n, j+1}(v)\right),
\end{gathered}
$$

and

$$
u_{a}(w)=u_{a}(v)
$$

for $a \neq j, j+1$.

Let $w_{0}, w_{1}$ be monomials such that

$$
\left(\omega_{0}\left(w_{0}\right), \ldots, \omega_{j-1}\left(w_{0}\right), \omega_{j}\left(w_{0}\right), \omega_{j+1}\left(w_{0}\right), \ldots\right)=\left(\omega_{0}(w), \ldots, \omega_{j-1}(w), 0,0, \ldots\right)
$$

and

$$
\left(\omega_{0}\left(w_{1}\right), \omega_{1}\left(w_{1}\right), \ldots\right)=\left(\omega_{j}(w), \omega_{j+1}(w), \ldots\right)
$$

Then, we have

$$
w=w_{0}\left(w_{1}\right)^{2^{j}}
$$

and, by the Cartan formula, we have

$$
\operatorname{Sq}^{2^{j}}(w)=\sum_{a+b=2^{j}} \operatorname{Sq}^{a}\left(w_{0}\right) \operatorname{Sq}^{b}\left(w_{1}^{2^{j}}\right) .
$$

Furthermore, by the Cartan formula, for $0<b<2^{j}$, we have

$$
\operatorname{Sq}^{b}\left(w_{1}^{2^{j}}\right)=0
$$

and for $b=2^{j}$, we have

$$
\operatorname{Sq}^{b}\left(w_{1}^{2^{j}}\right)=\left(\operatorname{Sq}^{1}\left(w_{1}\right)\right)^{2^{j}}
$$

So, we have

$$
\mathrm{Sq}^{2^{j}}(w)=w_{0}\left(\mathrm{Sq}^{1}\left(w_{1}\right)\right)^{2^{j}}+\left(\mathrm{Sq}^{2^{j}}\left(w_{0}\right)\right) w_{1}^{2^{j}} .
$$

For $a>0$ and a monomial $w^{\prime}, \mathrm{Sq}^{a}\left(w^{\prime}\right)$ is a linear combination of monomials $w^{\prime \prime}$ such that

$$
\left.\left.\left(\omega_{0}\left(w^{\prime \prime}\right), \omega_{1}\left(w^{\prime \prime}\right), \ldots\right)<\left(\omega_{0}\right) w^{\prime}\right), \omega_{1}\left(w^{\prime}\right), \ldots\right)
$$

Hence, we have

$$
\operatorname{Sq}^{2^{j}}(w) \equiv w_{0}\left(\operatorname{Sq}^{1}\left(w_{1}\right)\right)^{2^{j}} \equiv v+v^{\prime} \bmod F_{n}^{\prime},
$$

where $F_{n}^{\prime}$ is the subspace spanned by monomials $v^{\prime \prime}$ such that

$$
\left(\omega_{0}\left(v^{\prime \prime}\right), \ldots, \omega_{k-1}\left(v^{\prime \prime}\right)\right)<(n-1, \ldots, n-1) .
$$

Therefore, we have the desired result.

Thus, for each monomial $v$ in $P_{n}^{d_{1}}$ such that 


$$
\left(\omega_{0}(v), \ldots, \omega_{k-1}(v)\right)=(n-1, \ldots, n-1),
$$

there exists a monomial $v^{\prime}$ such that

$$
\begin{gathered}
\left(\omega_{0}\left(v^{\prime}\right), \ldots, \omega_{k-1}\left(v^{\prime}\right)\right)=(n-1, \ldots, n-1), \\
u_{0}\left(v^{\prime}\right) \geqslant u_{1}\left(v^{\prime}\right) \cdots \geqslant u_{k-1}\left(v^{\prime}\right)
\end{gathered}
$$

and

$$
v \equiv v^{\prime} \bmod F_{n} .
$$

Lemma 3.4. Let $v$ be a monomial such that

$$
\left(\omega_{0}(v), \ldots, \omega_{k-1}(v)\right)=(n-1, \ldots, n-1) .
$$

Suppose that

$$
u_{j}(v)=u_{j+1}(v)>u_{j+2}(v) .
$$

Let $v^{\prime}$ be the unique monomial such that $u_{a}(v)=u_{a}(v)$ for $a \neq j, j+1, j+2$ and $u_{j}\left(v^{\prime}\right)>u_{j+1}\left(v^{\prime}\right)=u_{j+2}\left(v^{\prime}\right), u_{j}\left(v^{\prime}\right)=u_{j}(v), u_{j+2}\left(v^{\prime}\right)=u_{j+2}(v)$. Then, $v \equiv v^{\prime}$ modulo $F_{n}$.

Proof. Let $w$ be the unique monomial such that

$$
\begin{gathered}
u_{j}(w)=u_{j+1}(w)=(1, \ldots, 1), \\
u_{j+2}(w)=\left(\alpha_{1, j}(v) \alpha_{1, j+2}(v), \ldots, \alpha_{n, j}(v) \alpha_{n, j+2}(v)\right),
\end{gathered}
$$

and

$$
u_{a}(w)=u_{a}(v)
$$

for $a \neq j, j+1, j+2$. Let $v^{\prime \prime}$ be the unique monomial such that

$$
\begin{gathered}
u_{j}\left(v^{\prime \prime}\right)=u_{j+1}\left(v^{\prime \prime}\right)<u_{j+2}\left(v^{\prime \prime}\right), \\
u_{j}\left(v^{\prime \prime}\right)=u_{j+2}(v), u_{j+2}\left(v^{\prime \prime}\right)=u_{j}(v),
\end{gathered}
$$

and

$$
u_{a}\left(v^{\prime \prime}\right)=u_{a}(v)
$$

for $a \neq j, j+1, j+2$. Then, as in the proof of Lemma 3.3, we have

$$
\mathrm{Sq}^{2^{j}} w \equiv v+v^{\prime \prime} \bmod F_{n}^{\prime}
$$

where $F_{n}^{\prime}$ is the subspace used in the proof of Lemma 3.3. Hence, we have

$$
v \equiv v^{\prime \prime} \bmod F_{n} .
$$

By applying Lemma 3.3 repeatedly, we have

$$
v^{\prime \prime} \equiv v^{\prime} \bmod F_{n}
$$


Thus, by Lemmas 3.3 and 3.4, for each monomial $v$ in $P_{n}^{d_{1}}$ such that

$$
\left(\omega_{0}(v), \ldots, \omega_{k-1}(v)\right)=(n-1, \ldots, n-1),
$$

there exists a monomial $v^{\prime}$ such that

$$
\begin{gathered}
\left(\omega_{0}\left(v^{\prime}\right), \ldots, \omega_{k-1}\left(v^{\prime}\right)\right)=(n-1, \ldots, n-1), \\
u_{0}\left(v^{\prime}\right)>u_{1}\left(v^{\prime}\right) \cdots>u_{\ell}\left(v^{\prime}\right)=\cdots=u_{k-1}\left(v^{\prime}\right)
\end{gathered}
$$

and

$$
v \equiv v^{\prime} \bmod F_{n} .
$$

In other words, there exist $1 \leqslant \ell \leqslant \min \{k, n\}$ and $\sigma \in \operatorname{Mono}(\ell)$ such that

$$
v \equiv \sigma\left(v_{\ell}\right) \bmod F_{n},
$$

where $\alpha_{i}(v)<k$ for $i \in\{\sigma(1), \ldots, \sigma(\ell)\}$ and $\alpha_{i}(v)=k$ for $i \notin\{\sigma(1), \ldots, \sigma(\ell)\}$.

Next, we prove that

$$
\left\{\pi\left(\sigma\left(v_{\ell}\right)\right) \mid 1 \leqslant \ell \leqslant \min \{k, n\}, \sigma \in \operatorname{Mono}(\ell)\right\}
$$

is linearly independent. Let $\lambda(\sigma): P_{n} \rightarrow P_{n-1}$ be a ring homomorphism defined by

$$
\begin{array}{ll}
\lambda(\sigma)\left(x_{i}\right)=x_{i} & \text { for } i<\sigma(\ell), \\
\lambda(\sigma)\left(x_{i}\right)=\sigma\left(x_{1}\right)+\cdots+\sigma\left(x_{\ell-1}\right) & \text { for } i=\sigma(\ell), \\
\lambda(\sigma)\left(x_{i}\right)=x_{i-1} & \text { for } i>\sigma(\ell) .
\end{array}
$$

Let us write

$$
\tilde{u}_{j}(v)=x_{1}^{\alpha_{1 j}(v)} \cdots x_{n}^{\alpha_{n j}(v)}
$$

Then, we have

$$
v=\prod_{j=0}^{\infty}\left(\tilde{u}_{j}(v)\right)^{2^{j}}
$$

It is clear that

$$
\lambda(\sigma)(v)=\prod_{j=0}^{\infty}\left(\lambda(\sigma)\left(\tilde{u}_{j}(v)\right)\right)^{2^{j}}
$$

and

$$
\lambda(\sigma)\left(x_{1} \cdots \widehat{x}_{a} \cdots x_{n}\right)= \begin{cases}x_{1} \cdots x_{n-1}+\sum v^{\prime} & \text { if } a \in\{\sigma(1), \ldots, \sigma(\ell)\}, \\ \sum v^{\prime} & \text { otherwise, }\end{cases}
$$

where $x_{1} \cdots \widehat{x}_{a} \cdots x_{n}$ is the monomial of degree $n-1$ obtained from $x_{1} \cdots x_{n}$ by removing $x_{a}$ and $\sum v^{\prime}$ indicates a linear combination of monomials $v^{\prime}$ such that $\omega_{0}\left(v^{\prime}\right)<n-1$. Therefore, it is easy to see that the following Lemma 3.5 holds.

Lemma 3.5. Suppose $1 \leqslant \ell, m \leqslant \min \{k, n\}, \sigma \in \operatorname{Mono}(m)$ and $\tau \in \operatorname{Mono}(\ell)$. Then,

$$
\lambda(\sigma)\left(\tau\left(v_{\ell}\right)\right) \equiv x_{1}^{2^{k}-1} \cdots x_{n-1}^{2^{k}-1} \not \equiv 0 \quad \bmod F_{n}
$$

if and only if

$$
\{\sigma(1), \ldots, \sigma(m)\} \supseteq\{\tau(1), \ldots, \tau(\ell)\},
$$

where $v_{\ell}$ is the monomial $v_{\ell}$ in Proposition 3.2. 
It follows from Lemma 3.5 that the linear map

$$
\lambda:\left(P_{n} / F_{n}\right)^{d_{1}} \rightarrow \prod_{1 \leqslant \ell \leqslant \min \{k, n\}}\left(\prod_{\sigma \in \operatorname{Mono}(\ell)}\left(P_{n-1} / F_{n-1}\right)^{d_{1}}\right)
$$

sending $\pi(v)$ to $(\pi(\lambda(\sigma)(v)))$ is an isomorphism. Thus,

$$
\left\{\pi\left(\sigma\left(v_{\ell}\right)\right) \mid 1 \leqslant \ell \leqslant \min \{k, n\}, \sigma \in \operatorname{Mono}(\ell)\right\}
$$

is a basis for $\left(P_{n} / F_{n}\right)^{d_{1}}$.

\section{The motivic hit problem}

In this section, we give the details of motivic hit problem. Let

$$
N_{n}=M_{n} /(\tau)=\Lambda_{n}\left(x_{1}, \ldots, x_{n}\right) \otimes \mathbb{Z} / 2\left[y_{1}, \ldots, y_{n}\right]
$$

and

$$
\mathcal{A}^{\prime}=\mathcal{A}^{*, *} /(\tau)
$$

Then, $N_{n}$ is an $\mathcal{A}^{\prime}$-module. Let $N_{n}^{d, *}$ be the subspace of $N_{n}$ spanned by elements of degree $(d, *)$. From now on, for the sake of simplicity, we say an element is of degree $d$ if its degree is $(d, *)$. Let $\mathcal{A}_{+}^{\prime}$ be the subset of $\mathcal{A}^{\prime}$ consisting of positive degree elements in $\mathcal{A}^{\prime}$ and

$$
\mathcal{A}_{+}^{\prime}\left(N_{n}\right)=\left\{a x \mid a \in \mathcal{A}_{+}^{\prime}, x \in N_{n}\right\} .
$$

Then, it is easy to see that $Q M_{n}^{d, *}$ in Section 1 is isomorphic to

$$
N_{n}^{d, *} /\left(\mathcal{A}_{+}^{\prime}\left(N_{n}\right) \cap N_{n}^{d, *}\right) .
$$

We consider the counterpart of $F_{n}$ in $N_{n}$. For the sake of notational simplicity, we write $\Lambda_{n}, Y_{n}$ for $\Lambda_{n}\left(x_{1}, \ldots, x_{n}\right), \mathbb{Z} / 2\left[y_{1}, \ldots, y_{n}\right]$, respectively. We denote by $\Lambda_{n}^{a}$, $Y_{n}^{2 b}$ the subspaces of $\Lambda_{n}, Y_{n}$ spanned by elements of degree $a, 2 b$, respectively. For a monomial

$$
z=x_{1}^{\varepsilon_{1}} \cdots x_{n}^{\varepsilon_{n}} y_{1}^{e_{1}} \cdots y_{n}^{e_{n}}
$$

in $N_{n}^{d, *}$, let us define $\alpha_{i j}(z) \in\{0,1\}$ by

$$
\varepsilon_{i}+2 e_{i}=\sum_{j=0}^{\infty} \alpha_{i j}(z) 2^{j} .
$$

We define non-negative integers $\alpha_{i}(z), \omega_{j}(z)$ by

$$
\begin{aligned}
& \alpha_{i}(z)=\sum_{j=0}^{\infty} \alpha_{i j}(z), \\
& \omega_{j}(z)=\sum_{i=1}^{n} \alpha_{i j}(z),
\end{aligned}
$$

respectively. Let $\mathcal{P}$ be the subalgebra of $\mathcal{A}^{\prime}$ generated by reduced power operations $\wp^{1}, \wp^{2}, \wp^{3}, \ldots$ of degree $2,4,6, \ldots$ Let $\mathcal{P}_{+}$be the subset of $\mathcal{P}$ consisting of positive degree elements in $\mathcal{P}$. Let $G_{n}$ be the subspace spanned by 


$$
\mathcal{P}_{+}\left(Y_{n}\right)=\left\{a x \mid a \in \mathcal{P}_{+}, x \in Y_{n}\right\}
$$

and monomials $z$ such that

$$
\left(\omega_{1}(z), \ldots, \omega_{k}(z)\right)<(n-1, \ldots, n-1) .
$$

Then, $G_{n} \subset Y_{n}$ is the counterpart of $F_{n}$ in $Y_{n}$. The ring isomorphism $\psi: Y_{n} \rightarrow P_{n}$ sending $y_{i}$ to $x_{i}$ commutes with the action of $\mathcal{P}, \mathcal{A}$ in the sense that $\psi\left(\wp^{c} y\right)=\operatorname{Sq}^{c} \psi(y)$. Thus, it induces an isomorphism

$$
\psi:\left(Y_{n} / G_{n}\right)^{2 b} \rightarrow\left(P_{n} / F_{n}\right)^{b} .
$$

We use this isomorphism to identify $\left(Y_{n} / G_{n}\right)^{2 b}$ with $\left(P_{n} / F_{n}\right)^{b}$, so that we can apply results on $\left(P_{n} / F_{n}\right)^{d_{1}}$ in Section 3 for $\left(Y_{n} / G_{n}\right)^{2 d_{1}}$.

We denote the projection from $\Lambda_{n}^{a} \otimes Y_{n}^{2 b}$ to $\Lambda_{n}^{a} \otimes\left(Y_{n} / G_{n}\right)^{2 b}$ by

$$
\pi: \Lambda_{n}^{a} \otimes Y_{n}^{2 b} \rightarrow \Lambda_{n}^{a} \otimes\left(Y_{n} / G_{n}\right)^{2 b} .
$$

Let $H_{n}$ be the subspace of $N_{n}$ spanned by

$$
\mathcal{A}_{+}^{\prime}\left(N_{n}\right)
$$

and monomials $z$ such that

$$
\left(\omega_{0}(z), \omega_{1}(z), \ldots, \omega_{k}(z)\right)<(k, n-1, \ldots, n-1) .
$$

Then, $H_{n} \subset N_{n}$ is the counterpart of $F_{n} \subset P_{n}$ in $N_{n}$. Since $Q_{0}$ maps $\Lambda_{n}^{a+1} \otimes Y_{n}^{2(b-1)}$ to $\Lambda_{n}^{a} \otimes Y_{n}^{2 b}$ and, for $c>0, \wp^{c}$ acts trivially on $\Lambda_{n}$, we have the following direct sum decomposition

$$
\left(N_{n} / H_{n}\right)^{d, *}=\bigoplus_{a+2 b=d} \Lambda_{n}^{a} \otimes\left(Y_{n} / G_{n}\right)^{2 b} / \pi\left(Q_{0}\left(\Lambda_{n}^{a+1} \otimes Y_{n}^{2(b-1)}\right)\right) .
$$

We prove the following proposition using Propositions 3.1 and 3.2.

Proposition 4.1. Suppose that $d=k+2 d_{1}, d_{1}=(n-1)\left(2^{k}-1\right), 1 \leqslant k<n$. For each monomial $z$ in

$$
\Lambda_{n}^{k} \otimes Y_{n}^{2 d_{1}}
$$

$z \in \Lambda_{n}^{k} \otimes G_{n}^{2 d_{1}}$ or there exist unique $\ell$ in $\{1, \ldots, k\}, \sigma_{1} \in \operatorname{Mono}(k)$ and $\sigma_{2} \in \operatorname{Mono}(\ell)$ such that

$$
z \equiv \sigma_{1}\left(x_{1} \cdots x_{k}\right) \sigma_{2}\left(\psi^{-1}\left(v_{\ell}\right)\right) \quad \bmod \Lambda_{n}^{k} \otimes G_{n}^{2 d_{1}},
$$

where $v_{\ell}$ is the monomial $v_{\ell} \in P_{n}^{d_{1}}$ in Proposition 3.2.

Proof. Suppose that $z=x_{i_{1}} \cdots x_{i_{k}} \otimes v$ and that $z$ is not in $\Lambda^{k} \otimes G_{n}^{2 d_{1}}$. Then by Proposition 3.1, we have

$$
\left(\omega_{0}(\psi(v)), \ldots, \omega_{k-1}(\psi(v))\right)=(n-1, \ldots, n-1) .
$$

So, by Proposition 3.2, there exists the unique pair $\left(\ell, \sigma_{2}\right)$ such that

$$
\psi(v) \equiv \sigma_{2}\left(v_{\ell}\right) \quad \bmod F_{n}
$$

in $P_{n}^{d_{1}}$. Let us define $\sigma_{1} \in \operatorname{Mono}(k)$ by $\sigma_{1}(j)=i_{j}$. Then, we have

$$
z \equiv \sigma_{1}\left(x_{1} \cdots x_{k}\right) \sigma_{2}\left(\psi^{-1}\left(v_{\ell}\right)\right) \quad \bmod \Lambda_{n}^{k} \otimes G_{n}^{2 d_{1}},
$$

as desired. 
Let $\mathcal{M}_{0}$ be the set of monomials $z=\sigma_{1}\left(x_{1} \cdots x_{k}\right) \sigma_{2}\left(\psi^{-1}\left(v_{\ell}\right)\right)$ in $\Lambda_{n}^{k} \otimes Y_{n}^{2 d_{1}}$ such that $\alpha_{i}(z)<k$ for some $i$ and $\mathcal{M}_{1}$ the set of monomials $z=\sigma_{1}\left(x_{1} \cdots x_{k}\right) \sigma_{2}\left(\psi^{-1}\left(v_{\ell}\right)\right)$ in $\Lambda_{n}^{k} \otimes Y_{n}^{2 d_{1}}$ such that $\alpha_{i}(z)=k$ for all $i$. In Section 3, we proved that

$$
\left\{\pi\left(\sigma_{2}\left(v_{\ell}\right)\right) \mid 1 \leqslant \ell \leqslant k, \sigma_{2} \in \operatorname{Mono}(\ell)\right\}
$$

is a basis for $\left(P_{n} / F_{n}\right)^{d_{1}}$, Since

$$
\left\{\sigma_{1}\left(x_{1} \cdots x_{k}\right) \mid \sigma_{1} \in \operatorname{Mono}(k)\right\}
$$

is a basis for $\Lambda_{n}^{k}, \pi\left(\mathcal{M}_{0} \cup \mathcal{M}_{1}\right)$ is a basis for $\Lambda_{n}^{k} \otimes\left(Y_{n} / G_{n}\right)^{2 d_{1}}$. If $\ell \neq k$, then $\alpha_{i}(z)<k$ for some $i$ in $\{1, \ldots, n\}$. If $\ell=k$ and $\sigma_{1} \neq \sigma_{2}$, then $\alpha_{i}(z)<k$ for some $i$ in $\{1, \ldots, n\}$. If $\ell=k$ and $\sigma_{1}=\sigma_{2}$, then

$$
v_{k}=x_{1}^{2^{k}-2^{k-1}-1} \cdots x_{k}^{2^{k}-2^{0}-1} x_{k+1}^{2^{k}-1} \cdots x_{n}^{2^{k}-1}
$$

and so

$$
x_{1} \cdots x_{k} \psi^{-1}\left(v_{k}\right)=x_{1} \cdots x_{k} y_{1}^{2^{k}-2^{k-1}-1} \cdots y_{k}^{2^{k}-2^{0}-1} y_{k+1}^{2^{k}-1} \cdots y_{n}^{2^{k}-1}
$$

is the monomial $z_{k}$ in Proposition 1.4. Therefore, we have

$$
\begin{aligned}
\mathcal{M}_{0}= & \left\{\sigma_{1}\left(x_{1} \cdots x_{k}\right) \sigma_{2}\left(\psi^{-1}\left(v_{\ell}\right)\right) \mid 1 \leqslant \ell<k, \sigma_{1} \in \operatorname{Mono}(k), \sigma_{2} \in \operatorname{Mono}(\ell)\right\} \\
& \cup\left\{\sigma_{1}\left(x_{1} \cdots x_{k}\right) \sigma_{2}\left(\psi^{-1}\left(v_{k}\right)\right) \mid \sigma_{1}, \sigma_{2} \in \operatorname{Mono}(k), \sigma_{1} \neq \sigma_{2}\right\}, \\
\mathcal{M}_{1}= & \left\{\sigma_{1}\left(x_{1} \cdots x_{k}\right) \sigma_{2}\left(\psi^{-1}\left(v_{k}\right)\right) \mid \sigma_{1}, \sigma_{2} \in \operatorname{Mono}(k), \sigma_{1}=\sigma_{2}\right\} \\
= & \left\{\sigma\left(z_{k}\right) \mid \sigma \in \operatorname{Mono}(k)\right\} .
\end{aligned}
$$

\section{Proof of Proposition 1.4}

Throughout this section, we suppose that $d=k+2 d_{1}, d_{1}=(n-1)\left(2^{k}-1\right), 1 \leqslant$ $k<n$. The algebra $\mathcal{A}^{\prime}$ is generated by $Q_{0}$ and the reduced power operations $\wp^{1}, \wp^{2}, \ldots$ By definition, we have $\mathcal{P}_{+}\left(Y_{n}\right) \subset G_{n}$. Moreover, $\wp^{a}(x)=0$ in $N_{n}$ for $a>0, x \in \Lambda_{n}$. Therefore, we have

$$
\pi\left(\wp^{a}(x \otimes y)\right)=\pi\left(x \otimes \wp^{a}(y)\right)=0,
$$

for each monomial $x \otimes y$ in $N_{n}^{d-2 a, *}$. So, we prove Proposition 1.4 by proving the following proposition.

Proposition 5.1. For each monomial $z$ in $N_{n}^{d-1}$ with $\omega_{0}(z)=k+1$,

$$
\pi\left(Q_{0}(z)\right) \in \Lambda_{n}^{k} \otimes\left(Y_{n} / G_{n}\right)^{2 d_{1}}
$$

is a linear combination of $\pi\left(z^{\prime}\right)\left(z^{\prime} \in \mathcal{M}_{0}\right)$ and $\pi\left(\sigma_{1}\left(z_{k}\right)+\sigma_{2}\left(z_{k}\right)\right)$, where $\sigma_{1}, \sigma_{2} \in$ $\operatorname{Mono}(k)$ and $z_{k}$ is the monomial $z_{k}$ in Proposition 1.4.

From Propositions 4.1 and 5.1, we have that $\pi\left(z_{k}\right)$ is not in

$$
\begin{aligned}
\pi\left(Q_{0}\left(\Lambda_{n}^{k+1} \otimes Y_{n}^{2\left(d_{1}-1\right)}\right)\right) & =\pi\left(Q_{0}\left(\Lambda_{n}^{k+1} \otimes Y_{n}^{2\left(d_{1}-1\right)}\right)\right)+\sum_{a>0} \pi\left(\wp^{a}\left(\Lambda_{n}^{k} \otimes Y_{n}^{2\left(d_{1}-a\right)}\right)\right. \\
& =\pi\left(\mathcal{A}_{+}^{\prime}\left(N_{n}\right)\right) .
\end{aligned}
$$

Thus, once we prove Proposition 5.1, we complete the proof of Proposition 1.4.

Let $z$ be a monomial in $\Lambda_{n}^{k+1} \otimes Y_{n}^{2\left(d_{1}-1\right)}$. 
If $\omega_{1}(z)=n-1$, then

$$
\begin{aligned}
\omega_{2}(z) \cdot 2^{2}+\omega_{3}(z) \cdot 2^{3}+\cdots & =d-1-(k+1)-2(n-1) \\
& =2 d_{1}-2 n \\
& =2(n-1)\left(2^{k}-1\right)-2 n \\
& =(n-1) 2^{k+1}+2 .
\end{aligned}
$$

Since $k \geqslant 1,(n-1) 2^{k+1}+2$ is not divisible by 4 but $\omega_{2}(z) \cdot 2^{2}+\omega_{3}(z) \cdot 2^{3}+\cdots$ is divisible by 4 . It is a contradiction. So, $\omega_{1}(z) \neq n-1$.

If $\omega_{1}(z)<n-2$, then $Q_{0}(z)$ is a linear combination of monomials $z^{\prime}$ such that $\omega_{0}\left(z^{\prime}\right)=k$ and $\omega_{1}\left(z^{\prime}\right)<n-1$. Hence, $Q_{0}(z) \in \Lambda_{n}^{k} \otimes G_{n}^{2 d_{1}}$.

There remain two cases: $\omega_{1}(z)=n$ or $n-2$. First, we deal with the case $\omega_{1}(z)=n$.

Proposition 5.2. Suppose that $z$ is a monomial in $N_{n}^{d-1}$ such that

$$
\left(\omega_{0}(z), \omega_{1}(z)\right)=(k+1, n) .
$$

Then, there exist monomials $z^{\prime}$ such that

$$
\left(\omega_{0}\left(z^{\prime}\right), \omega_{1}\left(z^{\prime}\right)\right)=(k+1, n-2)
$$

and

$$
Q_{0}(z)=Q_{0}\left(\sum z^{\prime}\right)
$$

Proof. Without loss of generality, we may assume that

$$
\begin{aligned}
& u_{0}(z)=(1, \ldots, 1,0,0, \ldots, 0), \\
& u_{1}(z)=(1, \ldots, 1,1,1, \ldots, 1) .
\end{aligned}
$$

Let $z^{\prime \prime}$ be a monomial such that

$$
\begin{aligned}
& u_{0}\left(z^{\prime \prime}\right)=(1, \ldots, 1,1,0, \ldots, 0), \\
& u_{1}\left(z^{\prime \prime}\right)=(1, \ldots, 1,0,1, \ldots, 1)
\end{aligned}
$$

and $u_{a}\left(z^{\prime \prime}\right)=u_{a}(z)$ for $a \geqslant 2$. Then,

$$
Q_{0}\left(z^{\prime \prime}\right)=z+\sum z^{\prime}
$$

where $\sum z^{\prime}$ is a linear combination of monomials $z^{\prime}$ such that $\left(\omega_{0}\left(z^{\prime}\right), \omega_{1}\left(z^{\prime}\right)\right)=(k+$ $1, n-2)$. Since $Q_{0} Q_{0}=0$, we have that

$$
Q_{0}(z)=Q_{0}\left(\sum z^{\prime}\right)
$$

as desired.

So, Proposition 5.3 below completes the proof of Proposition 5.1.

Proposition 5.3. Suppose that $z$ is a monomial in $N_{n}^{d-1}$ such that

$$
\left(\omega_{0}(z), \omega_{1}(z)\right)=(k+1, n-2) .
$$

If $\alpha_{i}(z)<k$ for some $i$, then $Q_{0}(z)$ is congruent to a linear combination of monomials in $\mathcal{M}_{0}$ modulo $\Lambda_{n}^{k} \otimes G_{n}^{2 d_{1}}$. If $\alpha_{i}(z)=k$ for all $i \in\{1, \ldots, n\}$, then

$$
Q_{0}(z) \equiv \sigma_{1}\left(z_{k}\right)+\sigma_{2}\left(z_{k}\right) \quad \bmod \Lambda_{n}^{k} \otimes G_{n}^{2 d_{1}} .
$$

Proof. In what follows, we consider everything modulo $\Lambda_{n}^{k} \otimes G_{n}^{2 d_{1}}$. The element $Q_{0}(z)$ 
is congruent to a linear combination of monomials $z^{\prime}$ such that $\alpha_{i}\left(z^{\prime}\right) \leqslant \alpha_{i}(z)$ for all $i \in\{1, \ldots, n\}$. Hence, if $\alpha_{i}(z)<k$ for some $i$, then $Q_{0}(z)$ is congruent to a linear combination of $z^{\prime}$ such that $\alpha_{i}\left(z^{\prime}\right)<k$. Hence, it is congruent to a linear combination of elements in $\mathcal{M}_{0}$.

If $\omega_{1}(z)=n-2$ and $\alpha_{i}(z)=k$ for all $i \in\{1, \ldots, n\}$, then without loss of generality, we may assume that

$$
\begin{aligned}
& u_{0}(z)=(1,1,1, \ldots, 1,0, \ldots, 0), \\
& u_{1}(z)=(0,0,1, \ldots, 1,1, \ldots, 1) .
\end{aligned}
$$

Then, $Q_{0}(z)$ is congruent to $\sigma_{1}\left(z_{k}\right)+\sigma_{2}\left(z_{k}\right)$ where $\sigma_{1}, \sigma_{2}$ in $\operatorname{Mono}(k), \sigma_{1}(1)=1$, $\sigma_{1}(2)=3, \ldots, \sigma_{1}(k)=k+1, \sigma_{2}(1)=2, \ldots, \sigma_{2}(k)=k+1$.

\section{References}

[1] S. Ault, Bott periodicity in the hit problem, Math. Proc. Cambridge Philos. Soc. 156 (2014), no. 3, 545-554, DOI 10.1017/S0305004114000085. MR3181639

[2] D. Dugger and D.C. Isaksen, The motivic Adams spectral sequence, Geom. Topol. 14 (2010), no. 2, 967-1014, DOI 10.2140/gt.2010.14.967. MR2629898

[3] N. Minami, The Adams spectral sequence and the triple transfer, Amer. J. Math. 117 (1995), no. 4, 965-985, DOI 10.2307/2374955. MR1342837

[4] D. Pengelley and F. Williams, Sparseness for the symmetric hit problem at all primes, Math. Proc. Cambridge Philos. Soc. 158 (2015), no. 2, 269-274, DOI 10.1017/S0305004114000668. MR3310245

[5] W.M. Singer, The transfer in homological algebra, Math. Z. 202 (1989), no. 4, 493-523, DOI 10.1007/BF01221587. MR1022818

[6] N. Sum, On the Peterson hit problem, Adv. Math. 274 (2015), 432-489, DOI 10.1016/j.aim.2015.01.010. MR3318156

[7] V. Voevodsky, Reduced power operations in motivic cohomology, Publ. Math. Inst. Hautes Études Sci. 98 (2003), 1-57, DOI 10.1007/s10240-003-0009-z. MR2031198

[8] G. Walker and R.M.W. Wood, Polynomials and the mod 2 Steenrod Algebra. Vol. 1. The Peterson Hit Problem, London Mathematical Society Lecture Note Series, vol. 441, Cambridge University Press, Cambridge, 2018. MR3729477

[9] R.M.W. Wood, Steenrod squares of polynomials and the Peterson conjecture, Math. Proc. Cambridge Philos. Soc. 105 (1989), no. 2, 307-309, DOI 10.1017/S0305004100067797. MR974986

[10] N. Yagita, Applications of Atiyah-Hirzebruch spectral sequences for motivic cobordism, Proc. Lond. Math. Soc. (3) 90 (2005), no. 3, 783-816, DOI 10.1112/S0024611504015084. MR2137831

Masaki Kameko kameko@shibaura-it.ac.jp

Department of Mathematical Sciences, Shibaura Institute of Technology, 307

Minuma-ku Fukasaku, Saitama-City 337-8570, Japan 